\title{
I Can C Clearly Now: How EPEC Inhibits Gut Vitamin C Transport by Dysregulating SVCT
}

\author{
Georgios I. Tsiaoussis ${ }^{1}$. Eirini Christaki ${ }^{2}$. Yiorgos Apidianakis ${ }^{3}$
}

Accepted: 27 August 2020 / Published online: 12 September 2020

(c) Springer Science+Business Media, LLC, part of Springer Nature 2020

Ascorbic acid (AA; vitamin C) is a natural water-soluble vitamin that is also a cofactor in numerous metabolic reactions catalyzed by $\mathrm{Cu}^{++}$dependent monooxygenases and $\mathrm{Fe}^{++}$dependent dioxygenases. Though many mammals are capable of de novo hepatic synthesis of ascorbate from glucose, humans and other primates cannot synthesize ascorbate due to an evolutionary mutation in the gene GULO, which normally encodes the enzyme L-gulono- $\gamma$-lactone oxidase, a catalyst of the last step of the biosynthetic pathway of AA [1].

AA is a potent antioxidant agent implicated in many biological functions, contributing to the inhibition of reactive oxygen species (ROS) production and the modulation of cytokines in the systemic inflammatory response syndrome. AA also enhances epithelial barrier function against pathogens by increasing the rate of collagen formation, accelerating tissue repair, and inducing the synthesis of key lipids and proteins. AA enhances resistance to infection by supporting both the innate and adaptive immune defense system, stimulates neutrophil migration to the site of infection, and promotes phagocytosis as well as the differentiation and maturation of B- and T-lymphocytes, particularly NK (natural killer) cells [2].

Although clinically observed deficiencies are rare, requirements may be increased and supplementation may be necessary for several conditions including alcoholism, cancer cachexia, chronic hemodialysis, prolonged diarrhea, gastrectomy, ileal resection, continuing infection, and intestinal diseases. Early manifestations of hypovitaminosis $\mathrm{C}$ include malaise, irritability, emotional disturbances,

Georgios I. Tsiaoussis

tsiaoussisgeorgios@yahoo.com

1 Department of Gastroenterology, Nicosia General Hospital, Nicosia, Cyprus

2 Medical School, University of Cyprus, Nicosia, Cyprus

3 Department of Biological Sciences, University of Cyprus, Nicosia, Cyprus arthralgia, hyperkeratosis of hair follicles, nosebleeds, and petechial hemorrhages. Severe vitamin $\mathrm{C}$ deficiency is a rare condition that leads to the development of scurvy [3].

Recent studies have demonstrated some effect of vitamin $\mathrm{C}$ administration on reducing mortality in patients with sepsis hospitalized in intensive care wards [4]. Given these data, and in light of the current COVID-19 pandemic, clinical trials have been initiated in order to investigate the therapeutic effects of vitamin $\mathrm{C}$ administration as an adjunct to conventional therapy in patients with COVID-19 pneumonia (NCT04323514).

Ascorbic acid is absorbed by the gut mucosa by active transport and by simple diffusion. Specific transport proteins mediate the transport of the oxidized form of vitamin $\mathrm{C}$, dehydroascorbic acid (DHA), and the reduced form, L-ascorbic acid, across biological membranes. Dehydroascorbic acid uptake is via the facilitated-diffusion glucose transporters, GLUT 1, 3, and 4, whereas L-ascorbic acid active transport is mediated via $\mathrm{Na}^{+}$-dependent systems via the membrane sodium vitamin $\mathrm{C}$ transporters (SVCTs). SVCT1, found in epithelial tissue, and SVCT2, found in most tissues except the lungs and skeletal muscles, import the reduced form of ascorbate across the basolateral plasma membrane of the intestinal cells [5]. The absorption of vitamin $\mathrm{C}$ and transport into the systematic circulation requires the transporters SVCT1 and SVCT2 in order to mediate electrogenic uptake of vitamin $\mathrm{C}$, using the sodium concentration gradient across the plasma membrane with a stoichiometry of $2 \mathrm{Na}^{+}$for each ascorbate. Human SVCT1 consists of 598 amino acids with a molecular mass of 64,831 Da. The SVCT2 peptide chain has a length of 650 amino acids with a molecular mass of 70,337 $\mathrm{Da}$ [6].

The solute carrier family 23 members SLC23A1 and SLC23A 2 comprise the protein coding genes. Single nucleotide polymorphisms (SNPs) of SLC23A1 and SLC23A2 have been described [7]. Furthermore, transcriptional and post-translational modifications (e.g., glycosylation and phosphorylation) of the SVCT proteins are likely involved 
in their regulation. Potential sites for phosphorylation by protein kinase $\mathrm{C}$ exist on the cytoplasmic surface of both proteins, with an additional protein kinase A site in SVCT1 [8]. Post-translational modifications for SVCT2 consist of phosphorylation at Ser70, Ser78, Ser81, Thr75, Thr79, Thr649, and glycosylation at Asn188 and Asn196 and, respectively, for SVCT1 phosphorylation at Ser593, Thr591, Thr596 and glycosylation at Asn138 and Asn144 (UniProtKB/Swiss-Prot).

Although intestinal absorption and transport of AA are multifactorial and affected by an interplay of external factors, mechanistic evidence is rudimentary. Several human diseases have been associated with polymorphisms and variations in the expression of SVCTs. However, the contribution of sodium:ascorbate cotransport to disease has not been fully elucidated. Furthermore, no studies to date have evaluated the consequence of enteropathogenic E. coli (EPEC) infection on the intestinal absorption of AA. In the study of Heskett and colleagues published in this issue of Digestive Diseases and Sciences, the authors utilized both in vivo and in vitro models in order to address this issue. The authors show for the first time the effect of EPEC infection on the expression of SVCTs [9].

The observed inhibitory effect of EPEC on AA uptake was accompanied by a dysregulated expression of SVCT1 and SVCT2 protein and mRNA both in vitro and in vivo. The reduction in SVCT1 and SVCT2 mRNA expression levels could be transcriptional, due to reduced expression, but also post-transcriptional via reduced mRNA stability. The authors showed that the expression levels of all three mature microRNA regulators of SVCT1 and SVCT2 (miR103a, miR141, and miR200a) were markedly up-regulated upon EPEC infection in Caco- 2 cells and mouse intestinal segments. These findings demonstrate that post-transcriptional mechanisms may act, at least in part, in the observed AA inhibitory effect in both model systems.

Moreover, Heskett and colleagues investigated the involvement of the accessory protein and positive regulator of SVCT1 function, glyoxylate reductase/hydroxypyruvate reductase (GRHPR) in facilitating the observed effect on intestinal uptake of vitamin $\mathrm{C}$. The authors demonstrate that EPEC infection may inhibit intestinal vitamin $\mathrm{C}$ absorption via post-transcriptional dysregulation of SVCT1 and SVCT2 expression by decreasing the expression of GRHPR in vitro and in vivo. Thus, GRHPR is part of the mechanism of hSVCT1inhibition upon EPEC infection and may also be partly responsible for mediating the observed AA malabsorption.

EPEC pathogenicity is mediated by attaching to intestinal epithelial cells and injecting virulence factors using a Type 3 secretion system (T3SS). As a result, host cells are subject to significant alterations in cytoskeletal networks, ion transport, inflammatory signaling, and tight junction function. Infection with $\Delta \operatorname{espA}, \Delta \operatorname{espB}$, and $\Delta$ espD mutants that totally lack T3SS function failed to inhibit AA uptake. On the other hand, infection with the EPEC-secreted effector mutants $\Delta \mathrm{espF}$ and $\Delta$ espG/G2, potentially retaining some T3SS activity, inhibited AA uptake. Thus, the respective proteins appear dispensable for the inhibitory effect. These observations indicate that the effect of EPEC on AA uptake in intestinal epithelia requires a functional T3SS and more than one of the factors it secretes [9].

This study is significant to the field of AA transport due to the insights it provides toward the nutritional management of patients with severe EPEC infection. Interventions to increase plasma ascorbate concentrations might be beneficial in patients with other severe bacterial or viral infections as well. An exogenous supply of the vitamin is one possible strategy that may reduce the deficit in intracellular ascorbate concentration following intestinal infection. Another potential therapeutic approach could be to enhance the activity of sodium-ascorbate cotransporters. One consideration though is the susceptibility of SVCT to downregulation by high supplementation of ascorbate since some interventional human clinical trials with vitamin $\mathrm{C}$ supplements failed to confer antioxidant protection or a clinical benefit [10]. Optimization of the regimen dosing may be essential to the success of future clinical studies using vitamin $\mathrm{C}$.

\section{References}

1. Nishikimi M, Fukuyama R, Minoshima S, Shimizu N, Yagi $\mathrm{K}$. Cloning and chromosomal mapping of the human nonfunctional gene for L-gulono-gamma-lactone oxidase, the enzyme for L-ascorbic acid biosynthesis missing in man. $J$ Biol Chem. 1994;269:13685-13688.

2. Carr AC, Maggini S. Vitamin C and Immune Function. Nutrients. 2017;9:1211.

3. Liang WJ, Johnson D, Jarvis SM. Vitamin C transport systems of mammalian cells. Mol Membr Biol. 2001;18:87-95.

4. Kuhn SO, Meissner K, Mayes LM, Bartels K. Vitamin C in sepsis. Curr Opin Anaesthesiol. 2018;31:55-60.

5. Rumsey SC, Kwon O, Xu GW, Burant CF, Simpson I, Levine M. Glucose transporter isoforms GLUT1 and GLUT3 transport dehydroascorbic acid. J Biol Chem. 1997;272:982-18989.

6. Savini I, Rossi A, Pierro C, Avigliano L, Catani MV. SVCT1 and SVCT2: key proteins for vitamin C uptake. Amino Acids. 2008;34:347-355.

7. Eck P, Erichsen HC, Taylor JG, et al. Comparison of the genomic structure and variation in the two human sodiumdependent vitamin C transporters, SLC23A1 and SLC23A2. Hum Genet. 2004;115:285-294.

8. Bürzle M, Suzuki Y, Ackermann D, et al. The sodiumdependent ascorbic acid transporter family SLC23. Mol Asp Med. 2013;34:436-454. 
9. Heskett CW, Teafatiller T, Hennessey C, et al. Enteropathogenic Escherichia coli infection inhibits intestinal ascorbic acid uptake via dysregulation of its transporter expression. Dig Dis Sci. (Epub ahead of print). https://doi.org/10.1007/s10620-02006389-x.

10. Wilson JX. Regulation of vitamin C transport. Annu Rev Nutr. 2005;25:105-125.
Publisher's Note Springer Nature remains neutral with regard to jurisdictional claims in published maps and institutional affiliations. 